\title{
Journal of Clinical Imaging Science
}

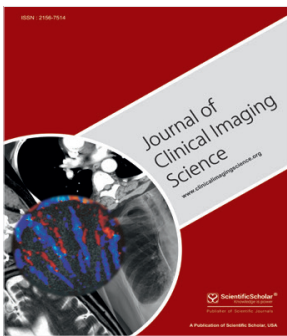

Case Report

\section{A Case of Ferumoxytol (Feraheme ${ }^{\circledR}$ ) Prompting Critical Modification to Our Patient Prebreast Magnetic Resonance Imaging Questionnaire}

\author{
Naziya Samreen, Asha A Bhatt, James Glockner, Christine U Lee \\ Department of Radiology, Mayo Clinic, Rochester, MN, USA.
}

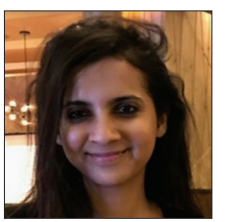

${ }^{*}$ Corresponding author:

Dr. Naziya Samreen,

Mayo Clinic, $2001^{\text {st }}$ Street SW,

Rochester, MN 55905, USA.

samreen.naziya@gmail.com

Received : 18 October 18

Accepted : 07 December 18

Published : 28 March 19

DOI

10.25259/JCIS-9-6

Quick Response Code:

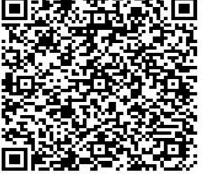

\begin{abstract}
Apparent gadolinium-based contrast enhancement was seen on precontrast-enhanced images on two breast magnetic resonance imaging (MRI) for a patient done 1 week apart. This was caused by ferumoxytol, which is used to treat some anemias. Such nongadolinium-based therapeutics exhibiting unintended MRI contrast-like properties preclude assessment of enhancement kinetics in breast MRI significantly limiting diagnostic interpretation. This case prompted a group discussion and subsequent modification to the patient breast MRI safety screening questionnaire at our institution.
\end{abstract}

Keywords: Artifact, contrast enhancement, ferumoxytol

\section{INTRODUCTION}

Since 2006, gadolinium-based contrast agents used in magnetic resonance imaging (MRI) have drawn much attention in both the medical and non-medical communities with regard to nephrogenic systemic fibrosis and more recently gadolinium deposition disease. As a result, strict screening is universally performed before any MRI with intravenous (IV) injection of gadolinium-based contrast agent. We report a case of an uncommon IV drug, ferumoxytol, which confounded a breast MRI examination performed for diagnostic purposes. Its associated T1 relativity resulted in a critical change to the breast MRI safety screening questionnaire.

\section{CASE REPORT}

A 29-year-old woman with an elevated risk of breast cancer (estimated lifetime risk 22\%) presented with persistent, intermittent, right milky nipple discharge and retroareolar pain.

The patient stopped nursing approximately 18 months before seeking medical attention. Relevant medical history included irregular menstrual cycles and iron deficiency anemia. After a negative mammographic and ultrasonographic diagnostic workup, breast MRI was performed.

Breast MRI was performed using a protocol that meets the standards of accreditation set forth by the American College of Radiology. ${ }^{[1]}$ There was apparent gadolinium-based contrast enhancement on precontrast imaging acquisitions [Figure 1a]. No significant increase in enhancement was noted following gadolinium administration [Figures $1 \mathrm{~b}$ and 2]. Similar findings were again noted on repeat MRI 1 week later.

This is an open-access article distributed under the terms of the Creative Commons Attribution-Non Commercial-Share Alike 4.0 License, which allows others to remix, tweak, and build upon the work non-commercially, as long as the author is credited and the new creations are licensed under the identical terms.

(-2019 Published by Scientific Scholar on behalf of Journal of Clinical Imaging Science 
After both examinations, an MRI technologist confirmed that precontrast images were indeed acquired before IV gadolinium-based contrast agent was appropriately administered. No IV infiltration of contrast was noted during contrast administration. The technologists did not notice anything unusual about the precontrast images and proceeded with gadolinium-based contrast injection without contacting a radiologist. A chart review showed that the patient's renal function was within normal limits. Her medication list in the electronic medical record only included cholecalciferol (Vitamin D3) and synthroid. On direct questioning, the patient mentioned that she had received an IV infusion of ferumoxytol (Feraheme ${ }^{\circledR}$ ) for her iron deficiency anemia. She received one dose a day before her first MRI and one dose a day before her repeat MRI.

The repeat MRI was interpreted despite lack of true precontrast imaging. Areas of apparent nonmass enhancement on the MRIs [Figure $1 \mathrm{a}$ and $\mathrm{b}$ ] were biopsied at the request of the patient and demonstrated benign pathology. Interestingly, no apparent enhancement on precontrast sequences was noted at the time of biopsy which was performed 3 weeks after the last ferumoxytol infusion. Our practice has since been modified to include IV iron supplementation in our patient questionnaire before performing a breast MRI.

\section{DISCUSSION}

Ferumoxytol is a carbohydrate-coated, superparamagnetic iron oxide nanoparticle with a plasma half-life of $15 \mathrm{~h}$. Following injection, the ferumoxytol nanoparticles are taken up by the reticuloendothelial system. The iron is released from the carbohydrate complex and is transferred to plasma transferrin or is stored in the liver, spleen, and bone marrow within the lysosomes. The carbohydrate coating is excreted by the kidneys and/or the gastrointestinal tract. ${ }^{[2]}$

Ferumoxytol is used as an IV iron supplement for the treatment of anemia, especially in patients with chronic kidney disease. ${ }^{[3]}$ Recommended dose is $510 \mathrm{mg}$ IV, followed by a second similar dose after 3-8 days. The most common adverse reactions include
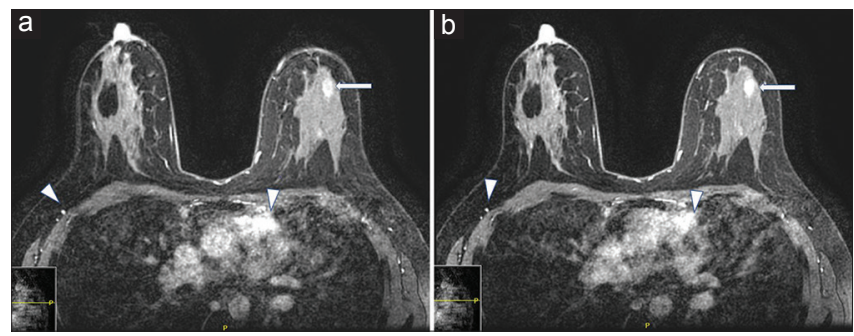

Figure 1: A 29-year-old female with ferumoxytol injection before breast magnetic resonance imaging. (a) Axial precontrast image demonstrating apparent enhancement in the heart and vasculature (arrowheads) and nonmass enhancement in the left breast (arrow). (b) Axial postcontrast image demonstrating similar enhancement in the heart and vasculature (arrowheads) and nonmass enhancement in the left breast (arrow). diarrhea, nausea, dizziness, hypotension, constipation, and peripheral edema, which can be seen in $\geq 2 \%$ of patients. ${ }^{[2]}$ One of the precautions of ferumoxytol is that it can interfere with MRI examinations. It can transiently alter the diagnostic ability of MRI for up to 3 months following the last dose with maximum alteration occurring within 1-2 days of administration. This is due to its intrinsic T1 shortening properties, which can produce a hyperintense signal on $\mathrm{T} 1$ sequences. In addition, it is also considered to be a negative contrast material due to its strong $\mathrm{T} 2{ }^{*}$ effect. ${ }^{[4]}$ It is recommended that T2 sequences not be performed earlier than 4 weeks after administration and T1 or proton-density sequences are recommended if MRI is required within 3 months of IV administration. ${ }^{[2]}$ Multiple artifacts have been noted in patients undergoing brain MRI within 3 days of receiving IV ferumoxytol, including susceptibility artifacts on diffusion-, gradient echo-, and susceptibility-weighted imaging. There was also noted decreased expected enhancement on postgadolinium contrast-enhanced T1 sequences. ${ }^{[3]}$ These phenomena have already been reported in the brain and abdomen, as well as MR angiography. ${ }^{[5,6]}$

Ferumoxytol is being investigated for its off-label use as an MRI contrast agent, predominantly for vascular imaging. It has demonstrated good visualization of cerebral vasculature in the early blood pool phase without obscuration of vessels. ${ }^{[7]}$ Multiple studies have also investigated its utility as a contrast agent in cardiac MRI. ${ }^{[8,9]}$ It has shown potential to be helpful for MRI in patients with chronic kidney disease. ${ }^{[10]}$

In this case, where two breast MRIs were performed without recognizing that the patient had recently received ferumoxytol before each MRI, we have changed our practice to include the use of IV iron supplementation such as ferumoxytol as part of our patient pre-MRI questionnaire [Figure 3]. To our knowledge, there is no other medication that causes such confounding effects on MRI.

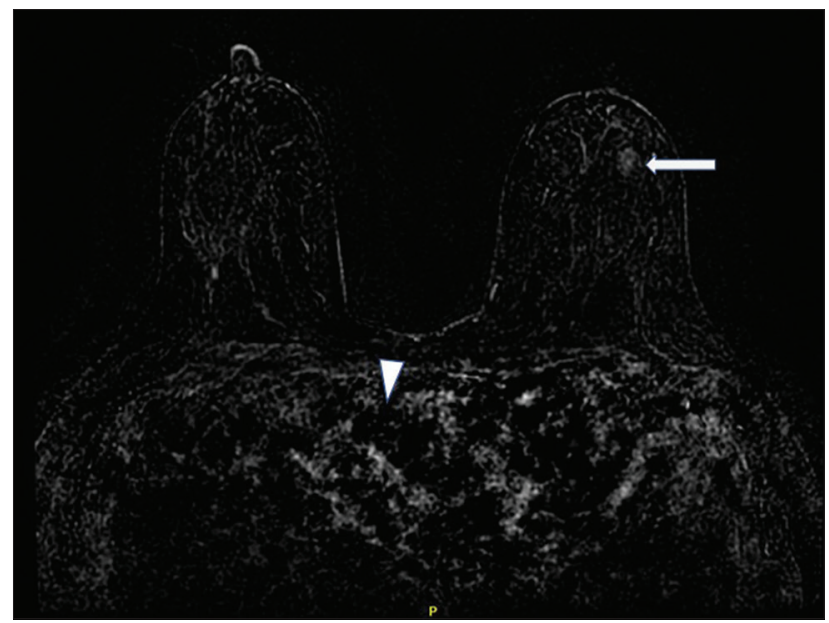

Figure 2: A 29-year-old female with ferumoxytol injection before breast magnetic resonance imaging. Subtraction image is extremely limited with no significant contrast noted in the heart and vasculature (arrowheads). Minimal nonmass enhancement is noted in the left breast (arrow). 


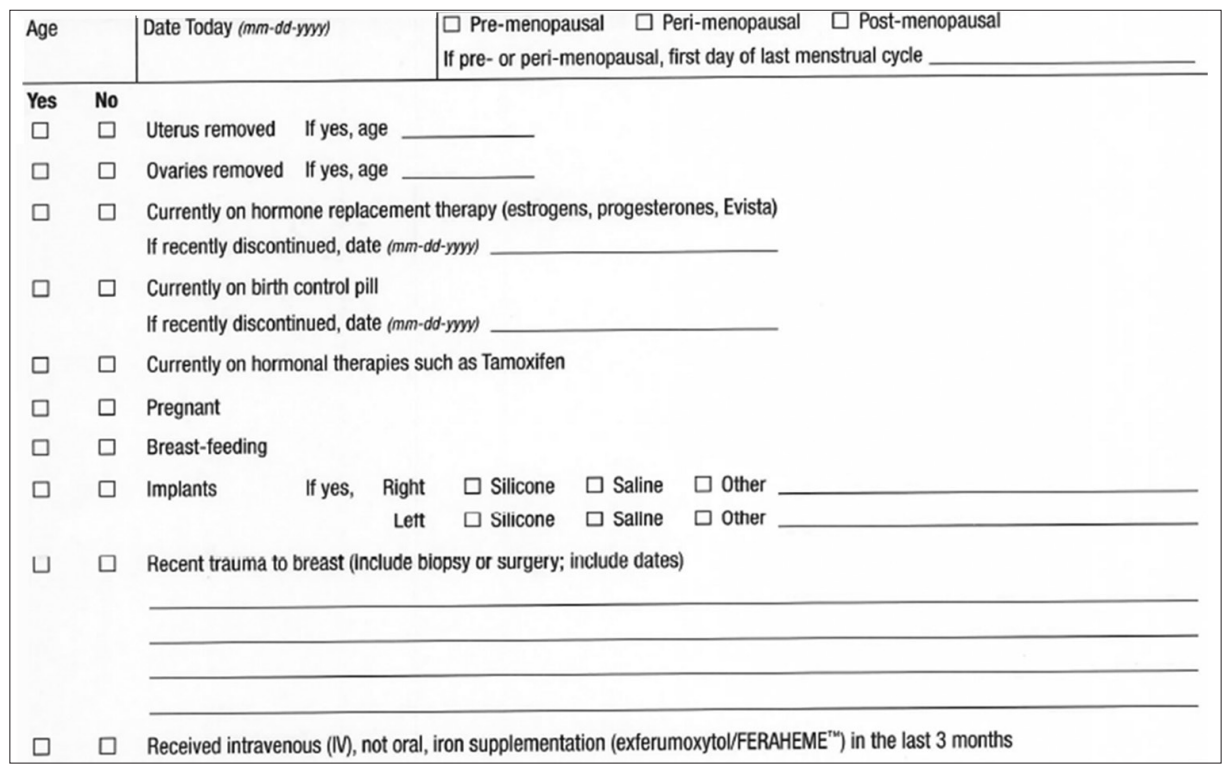

Figure 3: Our modified breast magnetic resonance imaging questionnaire includes a question about administration of FERAHEME as noted in the last sentence.

\section{CONCLUSION}

Dynamic contrast-enhanced MRI examinations with gadolinium-based contrast agents are not without risks, especially as we continue to learn more about nephrogenic systemic fibrosis and gadolinium deposition in the brain. As such, obtaining an optimal diagnostic quality MRI while minimizing the unnecessary use of gadolinium is of utmost importance. This case serves to emphasize the need to check the medical record for possible medications, specifically IV iron supplementation that would interfere with the diagnostic quality of a breast MRI examination.

\section{Declaration of patient consent}

The authors certify that they have obtained all appropriate patient consent forms. In the form the patient(s) has/have given his/her/their consent for his/her/their images and other clinical information to be reported in the journal. The patients understand that their names and initials will not be published and due efforts will be made to conceal their identity, but anonymity cannot be guaranteed.

\section{Financial support and sponsorship}

Nil.

\section{Conflicts of interest}

There are no conflicts of interest.

\section{REFERENCES}

1. ACR Practice Parameter for the Performance of Contrast Enhanced Magnetic Resonance Imaging (MRI) of the Breast. Revised; 2018. Available from: https://www.acr.org/-/media/ACR/Files/
Practice-Parameters/mr-contrast-breast.pdf. [Last accessed on 2018 Apr 04].

2. Feraheme (Ferumoxytol) Injection Label. FDA; 2018. Available from: https://www.accessdata.fda.gov/drugsatfda_docs/label/2009/ 022180lbl.pdf. [Last accessed on 2018 Mar 31].

3. Bowser BA, Campeau NG, Carr CM, Diehn FE, McDonald JS, Miller GM, et al. Incidental ferumoxytol artifacts in clinical brain MR imaging. Neuroradiology 2016;58:1087-91.

4. Dósa E, Tuladhar S, Muldoon LL, Hamilton BE, Rooney WD, Neuwelt EA. MRI using ferumoxytol improves the visualization of central nervous system vascular malformations. Stroke 2011;42:1581-8.

5. Schieda N. Parenteral ferumoxytol interaction with magnetic resonance imaging: A case report, review of the literature and advisory warning. Insights Imaging 2013;4:509-12.

6. Ruangwattanapaisarn N, Hsiao A, Vasanawala SS. Ferumoxytol as an off-label contrast agent in body 3T MR angiography: A pilot study in children. Pediatr Radiol 2015;45:831-9.

7. Varallyay $\mathrm{P}$, et al. Visualisation of normal and abnormal brain vasculature with different ferumoxytol doses. J Clin Oncol 2017; 35:15 suppl:e13517-e13517.

8. Han F, Zhou Z, Han E, Gao Y, Nguyen KL, Finn JP, et al. Self-gated 4D multiphase, steady-state imaging with contrast enhancement (MUSIC) using rotating cartesian K-space (ROCK): Validation in children with congenital heart disease. Magn Reson Med 2017;78:472-83.

9. Piccini D, et al. Respiratory self navigated whole-heart angiography with ultra-small super-paramagnetic iron oxide particles: A feasibility study. Journal of Cardiovascular Magnetic Resonance 2016;18 Suppl 1:56. doi:10.1186/1532-429x-18-s1-p56.

10. Stoumpos S, Hennessy M, Vesey AT, Radjenovic A, Kasthuri R, Kingsmore DB, et al. Ferumoxytol-enhanced magnetic resonance angiography for the assessment of potential kidney transplant recipients. Eur Radiol 2018;28:115-23.

How to cite this article: Samreen N, Bhatt AA, Glockner J, Lee CU. A case of ferumoxytol (Feraheme ${ }^{\circledR}$ ) prompting critical modification to our patient prebreast magnetic resonance imaging questionnaire. J Clin Imaging Sci 2019;9:6. 